\title{
THE SUM-OF-DIGITS FUNCTION ON ARITHMETIC PROGRESSIONS
}

\author{
LUKAS SPIEGELHOFER AND THOMAS STOLL
}

\begin{abstract}
Let $s_{2}$ be the sum-of-digits function in base 2, which returns the number of non-zero binary digits of a nonnegative integer $n$. We study $s_{2}$ along arithmetic subsequences and show that - up to a shift - the set of $m$-tuples of integers that appear as an arithmetic subsequence of $s_{2}$ has full complexity.
\end{abstract}

\section{Results}

The binary sum-of-digits function $s_{2}$ is an elementary object studied in number theory. It is defined by the equation

$$
s_{2}\left(\varepsilon_{\nu} 2^{\nu}+\cdots+\varepsilon_{0} 2^{0}\right)=\varepsilon_{\nu}+\cdots+\varepsilon_{0},
$$

where $\varepsilon_{i} \in\{0,1\}$ for $0 \leq i \leq \nu$. Despite the simplicity of definition, the behaviour of $s_{2}$ on arithmetic progressions is not fully understood. Cusick's conjecture on the sum-ofdigits function [3, 9] concerns this area of research: for an integer $t \geq 0$, we define the limit

$$
c_{t}=\lim _{N \rightarrow \infty} \frac{1}{N}\left|\left\{n: 0 \leq n<N, s_{2}(n+t) \geq s_{2}(n)\right\}\right| .
$$

(The limit exists, see for example Bésineau [2]. In fact, the set in this definition is periodic with period $2^{k}$ for some $k$.) Cusick's conjecture states that

$$
c_{t}>1 / 2
$$

for all $t \geq 0$. Drmota, Kauers, and the first author [3] proved that $c_{t}>1 / 2$ for almost all $t$ in the sense of asymptotic density; we also wish to note the works by Emme and Prikhod'ko [6] and Emme and Hubert [4, 5], and the recent partial result by the first author [9].

In the current note, motivated by Cusick's conjecture, we are concerned with the $(m+1)$-tuple $\left(s_{2}(n), s_{2}(n+t), \ldots, s_{2}(n+m t)\right)$, where $t \geq 0$ and $m \geq 1$ are integers. We aim to understand the set of tuples that can occur, as $n$ and $t$ run. In fact, our theorem states that, up to a shift, all tuples occur.

Theorem 1.1. Assume that $k_{1}, \ldots, k_{m} \in \mathbb{Z}$. There exist $n$ and $t$ such that for $1 \leq \ell \leq$ $m$,

$$
k_{\ell}=s_{2}(n+\ell t)-s_{2}(n) .
$$

2010 Mathematics Subject Classification. 11A63, 11B25.

The authors acknowledge support by the project MuDeRa, which is a joint project between the FWF (Austrian Science Fund) and the ANR (Agence Nationale de la Recherche). Moreover, the first author was supported by the FWF project F5502-N26, which is a part of the Special Research Program "Quasi Monte Carlo methods: Theory and Applications"; the second author was supported by the project ANR-18-CE40-0018. 
This is a generalization of the statement that the Thue-Morse sequence $\mathbf{t}$ has full arithmetic complexity, meaning that every finite word $\omega \in\{0,1\}^{L}$ occurs as an arithmetic subsequence of $\mathbf{t}$. This was first proved in [1] and also follows from Müllner and the first named author [8, and Konieczny [7.

Theorem 1.1 is not hard to prove for $m=1$. We present three arguments leading to this fact.

(1) Assume first that $k \geq 0$. Set $n=2^{k+1}$ and $t=2^{k}-1$. Then $s_{2}(n+t)=k+1$ and $s_{2}(n)=1$, yielding $k=s_{2}(n+t)-s_{2}(n)$. If $k<0$, we set $n=2^{-k+1}-1$ and $t=1$. Then $s_{2}(n)=-k+1$ and $s_{2}(n+t)=1$, which yields $s_{2}(n+t)-s_{2}(n)=k$. Alternatively, we may also write, as in the case $m=2$ presented below, $t=2^{c}-1$ and $n=2^{c-1}\left(2^{a}-1\right)$, for positive integers $a$ and $c$. We obtain $s_{2}(n+t)=c$ and $s_{2}(n)=a$, and clearly the difference $c-a$ runs through all integers.

(2) We have $s_{2}(n+1)-s_{2}(n)=1-\nu_{2}(n+1) \leq 1$, where $\nu_{2}(m)=\max \{k \geq$ $\left.0: 2^{k} \mid m\right\}$ for $m \geq 1$ is the 2 -adic valuation of $m$. This formula follows by considering the number of $1 \mathrm{~s}$ with which the binary expansion of $n$ ends. Since $s_{2}\left(2^{\ell}\right)=1$ and $s_{2}\left(2^{\ell+1}-1\right)=\ell+1$, we obtain the fact that $s_{2}(n)$ attains all values in $\{1, \ldots, \ell+1\}$ as $n$ varies in $\left\{2^{\ell}, \ldots, 2^{\ell+1}-1\right\}$. Let $k \in \mathbb{Z}$ be given and set $\ell=2|k|$. Choose $n \in\left\{2^{\ell}, \ldots, 2^{\ell+1}-1\right\}$ such that $s_{2}(n)=|k|+1$ and $n^{\prime} \in\left\{2^{\ell+1}, \ldots, 2^{\ell+2}-1\right\}$ such that $s_{2}\left(n^{\prime}\right)=|k|+1+k$. Then $s_{2}\left(n^{\prime}\right)-s_{2}(n)=k$, which implies the statement.

(3) Consider the densities

$$
\delta(k, t)=\lim _{N \rightarrow \infty} \frac{1}{N}\left|\left\{n: 0 \leq n<N, s_{2}(n+t)-s_{2}(n)=k\right\}\right|
$$

(as it was the case for $c_{t}$, this asymptotic density exists [2]). These quantities satisfy the following recurrence [3]:

$$
\begin{aligned}
\delta(k, 1) & = \begin{cases}2^{k-2}, & k \leq 1 ; \\
0 & \text { otherwise; }\end{cases} \\
\delta(k, 2 t) & =\delta(k, t) ; \\
\delta(k, 2 t+1) & =\frac{1}{2} \delta(k-1, t)+\frac{1}{2} \delta(k+1, t+1) .
\end{aligned}
$$

From this, it is very easy to show that $\delta(k, t)>0$ for all $k \leq s_{2}(t)$. For $k$ given, choose $t$ in such a way that $s_{2}(t) \geq k$; the positivity of the density $\delta(k, t)$ implies that there exists an $n$ such that $s_{2}(n+t)-s_{2}(n)=k$.

For $m=2$, it is also possible to obtain the statement by elementary considerations: consider integers $a, c \geq 1, b, d \geq 0$ and choose the integers $n$ and $t$ in such a way that the binary expansions look as follows:

$$
\begin{aligned}
& n: \overbrace{1 \cdots 110 \cdots 0}^{a} \overbrace{1 \cdots 10 \cdots 0}^{b} 10 \\
& t: \quad \underbrace{1 \cdots 1}_{c} 0 \cdots 0 \underbrace{1 \cdots 1}_{d} .
\end{aligned}
$$

The sums of digits of $n, n+t$ and $n+2 t$ respectively are $a+b, b+c+d$ and $c+d$ respectively. By varying the variables, we can obtain the statement for all integers $k_{1}$ 
and $k_{2}$ such that $k_{2} \leq k_{1}$. For the case $k_{1}<k_{2}$, we use the following configuration of the integers $n$ and $t$, where $a, d \geq 1$ and $c \geq 0$ :

$$
n: \quad \overbrace{1 \cdots 110 \cdots 0}^{a} \begin{aligned}
& \underbrace{1 \cdots 1}_{c} 10 \cdots 0 \underbrace{11 \cdots 1}_{d} . \\
& 0 .
\end{aligned}
$$

The sums of digits of $n, n+t$ and $n+2 t$ are $a, d$ and $c+d$ respectively, and we see that we obtain all pairs $\left(k_{1}, k_{2}\right) \in \mathbb{Z}^{2}$ such that $k_{1} \leq k_{2}$.

However, the method quickly experiences difficulties, as multiplication by 3 is not a shift of the binary digits anymore. While we believe that the case $m=3$ can be made work by some effort, a general principle is not apparent. Therefore we choose a different approach.

We prove Theorem 1.1 by induction on $m$, the cases $m=1,2$ having been discussed above. Assume that $m \geq 3$ and let $k_{1}, \ldots, k_{m} \in \mathbb{Z}$ be given. By induction hypothesis, there exist $t_{0}$ and $n_{0}$ such that $k_{\ell}=s_{2}\left(n_{0}+\ell t_{0}\right)-s_{2}\left(n_{0}+(\ell-1) t_{0}\right)$ for $1 \leq \ell<m$. Set $k_{m}^{\prime}=s_{2}\left(n_{0}+m t_{0}\right)-s_{2}\left(n_{0}+(m-1) t_{0}\right)$. We are going to show that we may vary $k_{m}^{\prime}$ by steps of \pm 1 , thus yielding the full statement.

By concatenation of binary expansions, it is sufficient to show the following statement.

There exist $t_{1}, n_{1}$ such that $s_{2}\left(n_{1}+\ell t_{1}\right)-s_{2}\left(n_{1}+(\ell-1) t_{1}\right)=0$ for $1 \leq \ell<m$ and $s_{2}\left(n_{1}+m t_{1}\right)-s_{2}\left(n_{1}+(m-1) t_{1}\right)= \pm 1$.

This concatenation is straightforward and summarized in the following lemma, which we will also use again in a moment.

Lemma 1.2. Let $\ell \geq 1, m \geq 1, n_{0}, \ldots, n_{k-1}$ and $t_{0}, \ldots, t_{k-1}$ be nonnegative integers. There exist nonnegative integers $n$ and $t$ such that

$$
s_{2}(n+\ell t)-s_{2}(n+(\ell-1) t)=\sum_{0 \leq j<k}\left(s_{2}\left(n_{j}+\ell t_{j}\right)-s_{2}\left(n_{j}+(\ell-1) t_{j}\right)\right)
$$

for $1 \leq \ell \leq m$.

Proof. The base case $k=1$ is trivial; it is sufficient to prove the statement for $k=2$, the general case following easily from repeated application of this case.

Let $N$ be so large that $n_{0}+m t_{0}<2^{N}$, and set $n=2^{N} n_{1}+n_{0}$ and $t=2^{N} t_{1}+t_{0}$. Since no carry propagation between the digits below and above $N$ occurs, we can add up the contribution of the two blocks in order to yield the statement.

We reduce the problem further, using this block representation again: choose $t_{j}=1$ for all $0 \leq j<k$; it is sufficient to find a $k \geq 1$ and nonnegative integers $n_{j}$ for $0 \leq j<k$ such that

$$
\sum_{0 \leq j<k}\left(s_{2}\left(n_{j}+\ell\right)-s_{2}\left(n_{j}+\ell-1\right)\right)= \begin{cases}0, & \text { if } 1 \leq \ell<m \\ \pm 1, & \text { if } \ell=m .\end{cases}
$$

In order to show (1.3), we use the telescoping sum

$$
\sum_{a \leq j<a+2^{L}} g(j)=s_{2}\left(a+2^{L}\right)-s_{2}(a)=g\left(\left\lfloor a / 2^{L}\right\rfloor\right),
$$


where $g(j)=s_{2}(j+1)-s_{2}(j)$. This representation yields for $1 \leq \ell \leq m$, where $L$ is chosen such that $2^{L} \leq m<2^{L+1}$,

$$
\begin{aligned}
\sum_{2 \cdot 2^{L}-m+\ell \leq j<3 \cdot 2^{L}-m+\ell} g(j) & =g\left(2+\left\lfloor(-m+\ell) / 2^{L}\right\rfloor\right)= \begin{cases}g(1)=0, & \text { if } 1 \leq \ell<m ; \\
g(2)=1, & \text { if } \ell=m ;\end{cases} \\
\sum_{2^{L}-m+\ell \leq j<2 \cdot 2^{L}-m+\ell} g(j) & =g\left(1+\left\lfloor(-m+\ell) / 2^{L}\right\rfloor\right)= \begin{cases}g(0)=1, & \text { if } 1 \leq \ell<m ; \\
g(1)=0, & \text { if } \ell=m ;\end{cases} \\
\sum_{3 \cdot 2^{L+1}+\ell \leq j<4 \cdot 2^{L+1}+\ell} g(j) & =g(3)=-1 \text { for } 1 \leq \ell \leq m .
\end{aligned}
$$

The first of these three identities yields the "+"-part of (1.3) by choosing $k=2^{L}$ and $n_{j}=2 \cdot 2^{L}-m+j$ for $0 \leq j<k$.

The "-"-part is obtained from the second and third identities: by considering the disjoint union $J=\left[2^{L}-m, 2 \cdot 2^{L}-m\right) \cup\left[3 \cdot 2^{L+1}, 4 \cdot 2^{L+1}\right)$, we have

$$
\sum_{j \in J}\left(s_{2}(j+\ell)-s_{2}(j+\ell-1)\right)= \begin{cases}g(0)=0, & \text { if } 1 \leq \ell<m ; \\ g(1)=-1, & \text { if } \ell=m .\end{cases}
$$

The statement follows by merging the two intervals and choosing $n_{j}$ accordingly. This finishes the proof of our theorem.

\section{Possible extensions}

From our proof, it is possible to actually construct integers $n$ and $t$ such that $s_{2}(n+$ $\ell t)-s_{2}(n)=k_{\ell}$ for $1 \leq \ell \leq m$. In particular, this yields integers $n$ and $t$ such that $\mathbf{t}_{n+\ell t}=\omega_{\ell}$ for $1 \leq \ell \leq m$, where $\left(\omega_{1}, \ldots, \omega_{m}\right) \in\{0,1\}^{m}$ and $\mathbf{t}$ is the Thue-Morse sequence on $\{0,1\}$. (Note that we also used $\mathbf{t}(2 n+1)=1-\mathbf{t}(n)$.) This gives a constructive result concerning the problem of full arithmetic complexity of the ThueMorse sequence considered in [1, 7, 8].

As an extension of the presented line of research, we are interested in the proportion of cases in which $s_{2}(n+\ell t)-s_{2}(n)=k_{\ell}$ occurs (for $1 \leq \ell \leq m$ ). For this, we define more generally

$$
\delta(\mathbf{k}, \varepsilon, t)=\operatorname{dens}\left\{n: s_{2}\left(n+\ell t+\varepsilon_{\ell}\right)-s_{2}(n)=k_{\ell} \text { for } 1 \leq \ell \leq m\right\},
$$

where $\mathbf{k}=\left(k_{1}, \ldots, k_{m}\right) \in \mathbb{Z}^{m}$ and $\varepsilon=\left(\varepsilon_{1}, \ldots, \varepsilon_{m}\right) \in \mathbb{N}^{m}$. This generalizes the array $\delta$ defined before. As in the one-dimensional case, the densities in this definition actually exist, and they satisfy the following recurrence relation:

$$
\begin{aligned}
\delta(\mathbf{k}, \boldsymbol{\varepsilon}, 2 t) & =\frac{1}{2} \operatorname{dens}\left\{n: s\left(2 n+2 \ell t+\varepsilon_{\ell}\right)-s(2 n)=k_{\ell} \text { for } 1 \leq \ell \leq m\right\} \\
& +\frac{1}{2} \operatorname{dens}\left\{n: s\left(2 n+2 \ell t+\varepsilon_{\ell}+1\right)-s(2 n+1)=k_{\ell} \text { for } 1 \leq \ell \leq m\right\} \\
& =\frac{1}{2} \delta\left(\mathbf{k}^{\prime}, \boldsymbol{\varepsilon}^{\prime}, t\right)+\frac{1}{2} \delta\left(\mathbf{k}^{\prime \prime}, \boldsymbol{\varepsilon}^{\prime \prime}, t\right),
\end{aligned}
$$


where $k_{\ell}^{\prime}=k_{\ell}-\varepsilon_{\ell} \bmod 2, k_{\ell}^{\prime \prime}=k_{\ell}+1-\left(\varepsilon_{\ell}+1\right) \bmod 2, \varepsilon_{\ell}^{\prime}=\left\lfloor\varepsilon_{\ell} / 2\right\rfloor$ and $\varepsilon_{\ell}^{\prime \prime}=\left\lfloor\left(\varepsilon_{\ell}+1\right) / 2\right\rfloor$; moreover,

$$
\begin{aligned}
\delta(\mathbf{k}, \varepsilon, 2 t+1) & =\frac{1}{2} \operatorname{dens}\left\{n: s\left(2 n+2 \ell t+\varepsilon_{\ell}+\ell\right)-s(2 n)=k_{\ell} \text { for } 1 \leq \ell \leq m\right\} \\
& +\frac{1}{2} \operatorname{dens}\left\{n: s\left(2 n+2 \ell t+\varepsilon_{\ell}+\ell+1\right)-s(2 n+1)=k_{\ell} \text { for } 1 \leq \ell \leq m\right\} \\
& =\frac{1}{2} \delta\left(\mathbf{k}^{\prime}, \varepsilon^{\prime}, t\right)+\frac{1}{2} \delta\left(\mathbf{k}^{\prime \prime}, \varepsilon^{\prime \prime}, t\right),
\end{aligned}
$$

where $k_{\ell}^{\prime}=k_{\ell}-\left(\varepsilon_{\ell}+\ell\right) \bmod 2, k_{\ell}^{\prime \prime}=k_{\ell}+1-\left(\varepsilon_{\ell}+\ell+1\right) \bmod 2, \varepsilon_{\ell}^{\prime}=\left\lfloor\left(\varepsilon_{\ell}+\ell\right) / 2\right\rfloor$ and $\varepsilon_{\ell}^{\prime \prime}=\left\lfloor\left(\varepsilon_{\ell}+\ell+1\right) / 2\right\rfloor$. This recurrence is the reason for the introduction of $\varepsilon$.

This recurrence can be used to prove statements on the densities $\delta(\mathbf{k}, \varepsilon, t)$. On the one hand, we may ask for generalizations of Cusick's conjecture, relating the relative sizes of the values $s_{2}(n), s_{2}(n+t), \ldots, s_{2}(n+m t)$ to one another.

Problem 1. Consider generalizations of Cusick's conjecture, proving for example, for many $t$, that

$$
\operatorname{dens}\left\{n: s_{2}(n+t) \geq s_{2}(n) \text { and } s_{2}(n+2 t) \leq s_{2}(n+t)\right\}>1 / 4 .
$$

Moreover, show that the constant $1 / 4$ is optimal.

On the other hand, we could ask for the overall shape of the $m$-dimensional probability distribution defined by $\delta(\cdot, \varepsilon, t)$.

Problem 2. Prove a multidimensional generalization of the theorem by Emme and Hubert [4]: for most $t$, the densities $\operatorname{dens}\left\{n: s_{2}(n+\ell t)-s_{2}(n)=k_{\ell}\right.$ for $\left.1 \leq \ell \leq m\right\}$ should define a probability distribution that is close to a multivariate Gaussian law.

We expect that nontrivial statements on both Problem 1 and Problem 2, at least for small $m$, can be obtained by extending the study of moments set forward by Emme and Hubert [4]. The transition to arbitrary dimensions $m$ however will necessitate new ideas.

\section{REFERENCES}

[1] S. V. Avgustinovich, D. G. Fon-Der-Flanss, And A. E. Frid, Arithmetical complexity of infinite words, in Words, languages \& combinatorics, III (Kyoto, 2000), World Sci. Publ., River Edge, NJ, 2003, pp. 51-62.

[2] J. BÉsineau, Indépendance statistique d'ensembles liés à la fonction "somme des chiffres", Acta Arith., 20 (1972), pp. 401-416.

[3] M. Drmota, M. Kauers, And L. Spiegelhofer, On a Conjecture of Cusick Concerning the Sum of Digits of $n$ and $n+t$, SIAM J. Discrete Math., 30 (2016), pp. 621-649.

[4] J. Emme And P. Hubert, Central Limit Theorem for Probability Measures Defined by Sum-of-digits Function in Base 2, 2018. To appear in Annali della Scuola Normale Superiore.

[5] - Normal distribution of correlation measures of binary sum-of-digits functions, Preprint, 2018. http://arxiv.org/abs/1810.11234.

[6] J. Emme And A. Prikhod'ko, On the Asymptotic Behavior of Density of Sets Defined by Sum-ofdigits Function in Base 2, Integers, 17 (2017), pp. A58, 28.

[7] J. Konieczny, Gowers norms for the Thue-Morse and Rudin-Shapiro sequences, Preprint, 2017. http://arxiv.org/abs/1611.09985.

[8] C. Müllner and L. Spiegelhofer, Normality of the Thue-Morse sequence along Piatetski-Shapiro sequences, II, Israel J. Math., 220 (2017), pp. 691-738. 
[9] L. Spiegelhofer, Approaching Cusick's conjecture on the sum-of-digits function, 2019. Accepted for publication in Integers; http://arxiv.org/abs/1904.08646.

Institute of Discrete Mathematics and Geometry, Vienna University of Technology, Vienna, Austria

Institut Élie Cartan de Lorraine, Université de Lorraine, Vandeuvre-lès-Nancy, France 